\title{
Pressurized Liquid Extraction optimization from supercritical defatted olive pomace: a green and selective phenolic extraction process
}

Nikolaos Katsinas ${ }^{1,2}$, Andreia Bento da Silva ${ }^{3,4}$, Amalia Enríquez-de-Salamanca ${ }^{2,5}$, Naiara Fernández 6 , Maria Rosário Bronze ${ }^{3,6,7}$, Soraya Rodríguez-Rojo ${ }^{1 *}$

${ }^{1}$ High Pressure Processes Group, Dpt. of Chemical Engineering and Environmental Technology, School of Engineering, University of Valladolid (UVa), Dr. Mergelina str., 47011, Valladolid, Spain

${ }^{2}$ Institute of Applied Ophthalmobiology (IOBA), University of Valladolid (UVa), Campus Miguel Delibes, Paseo de Belén 17, 47011, Valladolid, Spain

${ }^{3}$ Faculty of Pharmacy, University of Lisbon (FFULisboa), Av. Prof. Gama Pinto, 1649-019, Lisbon, Portugal

${ }^{4}$ Faculdade de Ciências e Tecnologia da Universidade Nova de Lisboa (FCT NOVA), Largo da Torre, 2829-516, Caparica, Portugal

${ }^{5}$ Biomedical Research Networking Center in Bioengineering, Biomaterials and Nanomedicine (CIBERBBN), Av. Monforte de Lemos, 3-5, 28029, Madrid, Spain

${ }^{6}$ Instituto de Biologia Experimental e Tecnológica (iBET), Apartado 12, 2780-901, Oeiras, Portugal

${ }^{7}$ Instituto de Tecnologia Química e Biológica da Universidade Nova de Lisboa (ITQB NOVA), Av. da República, 2780-157, Oeiras, Portugal

Number of pages: 4

Number of Tables: 4

Number of Figures: 1

*Corresponding author: Soraya Rodríguez Rojo, E-mail: soraya.rodriguez@uva.es 
Table S1. ANOVA for each response. Significance $(p$-value $<0.05)$ is presented in bold.

\begin{tabular}{|c|c|c|c|c|c|c|c|c|c|c|c|c|}
\hline \multirow[b]{2}{*}{ Response } & \multirow[b]{2}{*}{ Model } & \multicolumn{11}{|c|}{ p-values } \\
\hline & & Model & $\begin{array}{l}\text { Lack of } \\
\text { fit test }\end{array}$ & $\mathbf{T}$ & EtOH\% & $S / L$ & $\mathrm{~T}^{2}$ & EtOH\% ${ }^{2}$ & $S / L^{2}$ & T-EtOH\% & EtOH\%-S/L & T-S/L \\
\hline EY & quadratic & $<0.0001$ & 0.5399 & $<0.0001$ & 0.0025 & $<0.0001$ & 0.0049 & 0.4469 & 0.1427 & 0.0001 & 0.5130 & 0.8825 \\
\hline AA & quadratic & $<0.0001$ & 0.1831 & 0.0065 & $<0.0001$ & 0.0151 & 0.0543 & 0.3516 & 0.0001 & 0.0004 & 0.0282 & 0.1024 \\
\hline TPC & quadratic & 0.0057 & 0.1906 & 0.6371 & 0.0016 & 0.2867 & 0.4443 & 0.4303 & 0.0010 & 0.0427 & 0.4518 & 0.4582 \\
\hline TFC & quadratic & $<0.0001$ & 0.1684 & $<0.0001$ & 0.2724 & 0.7155 & 0.0605 & 0.0678 & 0.0184 & 0.0047 & 0.1539 & 0.0005 \\
\hline $\mathrm{OL}$ & linear & $<0.0001$ & 0.4284 & $<0.0001$ & 0.0010 & 0.4284 & - & - & - & - & - & - \\
\hline 3,4-DHPEA-DEDA & quadratic & $<0.0001$ & 0.1468 & $<0.0001$ & $<0.0001$ & 0.0545 & 0.0007 & 0.6741 & 0.1428 & $<0.0001$ & 0.6039 & 0.0598 \\
\hline HT & quadratic & $<0.0001$ & 0.6793 & 0.0003 & 0.8065 & 0.0234 & 0.0814 & 0.2055 & 0.0001 & 0.0004 & 0.0149 & 0.3302 \\
\hline TY & quadratic & $<0.0001$ & 0.5205 & 0.4927 & 0.0163 & 0.0012 & 0.1769 & 0.2818 & 0.0105 & $<0.0001$ & 0.0686 & 0.0473 \\
\hline
\end{tabular}

Table S2. Equations describing each DoE response.

\begin{tabular}{|c|c|c|}
\hline $\begin{array}{l}\text { Equation } \\
\text { number }\end{array}$ & Response & Equation \\
\hline 1 & EY & $145.31000+(0.10264 \times \mathrm{xT})+(3.15069 \times \mathrm{xETOH} \%)-(435.50900 \times \mathrm{S} / \mathrm{L})+\left(0.00927 \times \mathrm{TT}^{2}\right)-(0.02876 \times \mathrm{T} \times \mathrm{EtOH} \%)$ \\
\hline 2 & AA & $\begin{array}{l}4.97029+(0.01298 \times T)-(0.01678 \times E t O H \%)-(3.93652 x S / L)+\left(13.02460 \times S / L^{2}\right)+(0.00072 \times T \times E t O H \%)- \\
(0.06713 \times E t O H \% x S / L)\end{array}$ \\
\hline 3 & TPC & $396.46900-(1.54001 \times E t O H \%)+\left(604.63400 x S / L^{2}\right)+(0.02121 \times T x E t O H \%)$ \\
\hline 4 & TFC & $6.67467+(0.10199 \times \mathrm{T})+\left(19.34900 \times \mathrm{S} / \mathrm{L}^{2}\right)+(0.00159 \times \mathrm{T} \times \mathrm{EtOH} \%)-(0.26530 \times \mathrm{TxS} / \mathrm{L})$ \\
\hline 5 & $\mathrm{OL}$ & $11.44810-(0.09217 \times \mathrm{T})+(0.07389 \times \mathrm{EtOH} \%)$ \\
\hline 6 & 3,4-DHPEA-DEDA & $45.50650+(0.02340 \times \mathrm{T})-(0.56711 \times \mathrm{EtOH} \%)-\left(0.00208 \times \mathrm{T}^{2}\right)+(0.00591 \times \mathrm{T} \times \mathrm{EtOH} \%)$ \\
\hline 7 & HT & $10.52700+(0.00611 \times T)-(20.66170 \times S / L)+\left(14.92940 \times S / L^{2}\right)+(0.00083 \times T \times E t O H \%)+(0.08830 x E t O H \% x S / L)$ \\
\hline 8 & TY & $8.40714-(0.10045 \times E t O H \%)-(7.89174 x \mathrm{~S} / \mathrm{L})+\left(4.30932 \times \mathrm{S} / \mathrm{L}^{2}\right)+(0.00070 \times \mathrm{TxEtOH} \%)+(0.02584 \times \mathrm{TxS} / \mathrm{L})$ \\
\hline
\end{tabular}

Table S3. Pearson correlation coefficient values among the DoE responses.

\begin{tabular}{|l|c|}
\hline & AA \\
\hline EY & $\mathbf{- 0 . 5 4 4 ^ { * }}$ \\
\hline AA & 1 \\
\hline TPC & $\mathbf{0 . 7 2 4 ^ { * * }}$ \\
\hline TFC & $\mathbf{0 . 5 6 1 ^ { * * }}$ \\
\hline OL & 0.311 \\
\hline 3,4-DHPEA-DEDA & $\mathbf{0 . 6 3 4 ^ { * * }}$ \\
\hline HT & 0.350 \\
\hline TY & 0.350 \\
\hline $\begin{array}{l}* \text { Correlation is significant at the } 0.05 \text { level (2-tailed) } \\
\text { ** Correlation is significant at the } 0.01 \text { level (2-tailed). }\end{array}$ \\
\hline
\end{tabular}


Table S4. Comparison between the predicted (PR) and the observed (OB) values for the DoE responses. Predicted values are accompanied by their $95 \%$ confidence interval. Run 1: $T=66.0^{\circ} \mathrm{C}, \mathrm{EtOH} \%=10.0 \%, \mathrm{~S} / \mathrm{L}=0.8 \mathrm{gOP} / \mathrm{mLSOLVENT}$. Run 2: $T=184.0^{\circ} \mathrm{C}$, EtOH\% $=90.0 \%, \mathrm{~S} / \mathrm{L}=0.8 \mathrm{gOP} / \mathrm{mLSOLVENT}$.

\begin{tabular}{|c|c|c|c|c|c|c|c|c|c|c|c|c|c|c|c|c|}
\hline \multirow[t]{2}{*}{ Run } & \multicolumn{2}{|c|}{$\begin{array}{c}\mathrm{EY} \\
\left(\mathrm{mg}_{\mathrm{DE}} / \mathrm{g}_{\mathrm{FD}-\mathrm{OP}-\mathrm{DO}}\right)\end{array}$} & \multicolumn{2}{|c|}{$\begin{array}{c}\mathrm{AA} \\
\left(\mathrm{mmol}_{\mathrm{TE}} / \mathrm{g}_{\mathrm{DE}}\right)\end{array}$} & \multicolumn{2}{|c|}{$\begin{array}{c}\text { TPC } \\
\left(\mathrm{mg}_{\mathrm{GAE}} / \mathrm{g}_{\mathrm{DE}}\right)\end{array}$} & \multicolumn{2}{|c|}{$\begin{array}{c}\text { TFC } \\
\left(\mathrm{mg}_{\mathrm{CATE}} / \mathrm{g}_{\mathrm{DE}}\right)\end{array}$} & \multicolumn{2}{|c|}{$\begin{array}{l}\text { OL richness } \\
\left(\mathrm{mg}_{\mathrm{oL}} / \mathrm{g}_{\mathrm{DE}}\right)\end{array}$} & \multicolumn{2}{|c|}{$\begin{array}{c}\text { 3,4-DHPEA- } \\
\text { DEDA richness } \\
\text { (mgoLE/gDE) }\end{array}$} & \multicolumn{2}{|c|}{$\begin{array}{l}\text { HT richness } \\
\left(\mathrm{mg}_{\mathrm{HT}} / \mathrm{g}_{\mathrm{DE}}\right)\end{array}$} & \multicolumn{2}{|c|}{$\begin{array}{l}\text { TY richness } \\
\left(\mathrm{mg}_{\mathrm{TY}} / \mathrm{g}_{\mathrm{DE}}\right)\end{array}$} \\
\hline & PR & OB & PR & OB & PR & OB & PR & OB & PR & OB & PR & OB & PR & OB & PR & $\mathrm{OB}$ \\
\hline 1 & $9 \pm 9$ & 8.0 & $\begin{array}{c}8.2 \pm \\
1.6\end{array}$ & 8.8 & $\begin{array}{c}248 \pm \\
42\end{array}$ & 282 & $23 \pm 4$ & 20 & $8 \pm 3$ & 6 & $52 \pm 10$ & 52 & $\begin{array}{c}4.0 \pm \\
1.9\end{array}$ & 4.4 & $\begin{array}{c}3.7 \pm \\
1.0\end{array}$ & 4.1 \\
\hline 2 & $49 \pm 9$ & 56.4 & $\begin{array}{c}6.2 \pm \\
1.6\end{array}$ & 5.1 & $\begin{array}{c}336 \pm \\
42\end{array}$ & 336 & $4 \pm 4$ & 6 & $3 \pm 3$ & 0 & $17 \pm 10$ & 9 & $\begin{array}{c}9.0 \pm \\
1.9\end{array}$ & 7.7 & $\begin{array}{c}5.0 \pm \\
1.0\end{array}$ & 4.1 \\
\hline
\end{tabular}



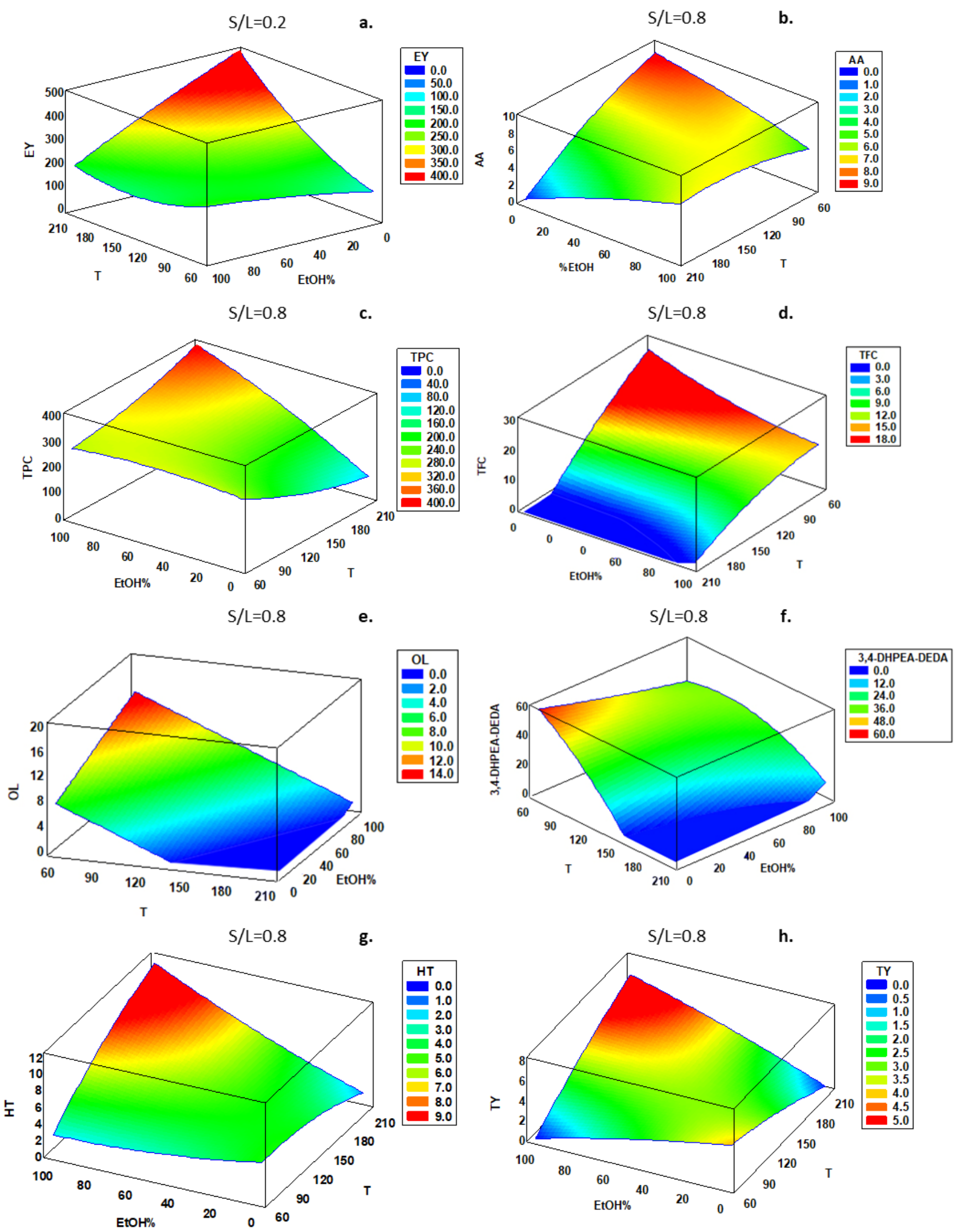

Figure S1. Response surface as a function of EtOH\% and T, for: a. EY, b. AA, c. TPC, d. TFC, e. OL richness, f. 3,4-DHPEA-DEDA richness, g. HT richness, $\boldsymbol{h}$. TY richness 